\title{
Flux erosion of magnetic clouds by reconnection with the Sun's open flux
}

\author{
Sanchita Pal ${ }^{1}$, Soumyaranjan Dash ${ }^{1}$, and Dibyendu Nandy ${ }^{1,2}$ \\ ${ }^{1}$ Center of Excellence in Space Sciences India, Indian Institute of Science Education and Research \\ Kolkata, Mohanpur 741246, West Bengal, India \\ ${ }^{2}$ Department of Physical Sciences, Indian Institute of Science Education and Research Kolkata, Mohanpur \\ 741246, West Bengal, India
}

\section{Key Points:}

- Flux of magnetic clouds is eroded due to magnetic reconnection with the solar open flux, impacting their geoeffectiveness in some cases.

- A linear relationship is observed between eroded and open flux with larger average flux erosion during the stronger cycle 23 compared to 24 .

- We establish a solar cycle link to the evolution of magnetic cloud flux during their interplanetary propagation.

\begin{abstract}
Magnetic clouds (MCs) are flux-rope magnetic structures forming a subset of solar coronal mass ejections which have significant space weather impacts. The geoeffectiveness of MCs depends on their properties which evolve during their interplanetary passage. Based on an analysis of observations spanning two solar cycles we establish that MCs interacting with the ambient solar wind magnetic field (i.e., heliospheric open flux) lose a substantial amount of their initial magnetic flux via magnetic reconnection, which in some cases, reduce their geoeffectiveness. We find a linear correlation between the eroded flux of MCs and solar open flux which is consistent with the scenario that MC erosion is mediated via the local heliospheric magnetic field draping around an MC during its interplanetary propagation. The solar open flux is governed by the sunspot cycle. This
\end{abstract}

Corresponding author: Dibyendu Nandy, dnandi@iiserkol.ac.in 
work therefore uncovers a hitherto unknown pathway for solar cycle modulation of the properties of MCs.

\section{Plain Language Summary}

Vast eruptions of magnetized plasma from the Sun known as coronal mass ejections (CMEs) are the main contributors to severe space weather conditions. The magnetic properties of CMEs - which determine their geoeffectiveness - evolve during their interplanetary journey. Therefore it is important to understand the physical processes that govern their evolution. Based on in situ satellite observations, and modeling here we provide evidence that the magnetic flux of CME associated magnetic clouds erode over their interplanetary journey due to reconnection with the ambient heliospheric open flux. We also demonstrate that this flux erosion impacts the geoeffectiveness of magnetic clouds in some cases. This mechanism establishes a novel connection between the solar cycle variation of the heliospheric open flux and space weather in the vicinity of solar system planets.

\section{Introduction}

Coronal mass ejections (CMEs) are magnetised plasma structures expelled sporadically from the Sun and travel toward the heliosphere as interplanetary coronal mass ejections (ICMEs). If an ICME has a smoothly rotating strong magnetic field and a low proton temperature (Burlaga et al., 1981; Klein \& Burlaga, 1982) it is categorised as a magnetic cloud (MC). The twisted flux ropes of MCs (Goldstein, 1983; Burlaga, 1988; Lepping et al., 1990) carry a significant amount of solar magnetic flux and helicity (Pal et al., 2017). While propagating through the interplanetary medium, the presence of a relative motion between an $\mathrm{MC}$ and the ambient plasma would result in a draping of the ambient interplanetary magnetic field (IMF) over the MC (Gosling \& McComas, 1987). Such draping has been directly observed (e.g., Crooker et al. (1985)) as well as modeled (e.g., Alksne and Webster (1970)). If the magnetic field orientation of the MC is opposite to that of the draped IMF, the process of magnetic reconnection (henceforth reconnection) is initiated (McComas et al., 1988). Due to this reconnection a part of the MC's magnetic flux is eroded away and an asymmetry is generated in its azimuthal magnetic flux that accumulates along the spacecraft trajectory. This fact has been established by observations (Dasso et al., 2006, 2007; Möstl et al., 2008; Ruffenach et al., 2012, 2015) 
as well as by using global MHD simulations (Schmidt \& Cargill, 2003; Taubenschuss et al., 2010). Lavraud et al. (2014) showed that erosion of an MC's magnetic flux may reduce its geoeffectiveness by a significant amount. Figure 1 shows an idealized schematic diagram of the draping of a purely radial ambient IMF about a fast moving MC and the MC's cross-section in a plane perpendicular to the ecliptic plane. The imbalance in the MC's accumulated azimuthal flux arises due to the reconnection at the MC's front.

An approximate understanding of the erosion of the magnetic flux of MCs due to reconnection with the IMF can be obtained with recourse to the Sweet-Parker model (Parker, 1957; Sweet, 1958). In the diffusion region (a small-scale region surrounding the reconnection site) Cassak and Shay (2007) showed that the reconnection rate scales as $E_{r e c} \sim$ $V_{1} B_{1} \sim V_{2} B_{2}$, where $V$ and $B$ are the plasma flow speed and magnetic field intensity, respectively, and the subscripts ' 1 ' and '2' refer to either side of the inflow region. For steady state reconnection $E_{r e c}$ is uniform at the reconnection region, where $E_{r e c}$ defines the rate of magnetic flux that is transferred from the inflow to the dissipation region (Nakamura et al., 2018). This transferred magnetic flux determines the amount of flux erosion $\left(F_{e r}\right)$ and one expects that $F_{e r}$ would scale linearly with the background IMF field strength (Cassak \& Shay, 2007).

The local Alfvén speed $\left(V_{A}\right)$ in the plasma near the reconnection site governs the reconnection rate (Parker, 1973; Birn et al., 2001; Cassak \& Shay, 2007). As $V_{A}$ decreases with the increasing heliospheric distance, the reconnection rate is expected to be higher closer to the Sun and in the inner heliosphere (e.g. Lavraud and Borovsky (2008)). Dasso et al. (2007) found an extended coherent magnetic region just behind an MC and interpreted that this region is formed due to the reconnection process which started $\approx 54$ hrs before the MC was observed. Lavraud et al. (2014) suggested that about $47 \%$ to $67 \%$ of the $\mathrm{MC}$ erosion is expected to occur within the orbit of Mercury $(\approx 0.39 \mathrm{AU}$ from the Sun). Ruffenach et al. (2015) found no correlation between the eroded amount of magnetic flux and the ambient solar wind properties observed in situ at 1 AU. The lack of correlation suggests that most of the reconnection is expected to occur during the MC's passage through the inner heliosphere and not at $1 \mathrm{AU}$.

Large scale open magnetic field originating from the solar corona extends out into the heliosphere and shapes the IMF. Utilizing the observed solar photospheric magnetic field information as boundary condition the heliospheric magnetic field can be reconstructed 
following the simplest assumption that the solar corona is current-free (Schatten et al., 1969). This approach is known as Potential Field Source Surface extrapolation (PFSS). In the outer radial boundary of the PFSS model the field lines are constrained to be radial which is consistent with the observations of the coronal hole foot-points (Wang et al., 1996). The open magnetic flux $\left(\phi_{\text {open }}\right)$ derived using the Sun's open magnetic field lines can be used to estimate the radial IMF intensity at any heliocentric distance from the Sun as the Ulysses spacecraft measurements show a latitudinal and longitudinal uniformity of the IMF's radial component (E. J. Smith \& Balogh, 2008; Balogh et al., 1995; Erdős \& Balogh, 2014). Therefore, the radial magnetic field intensity $\left(\left|B_{r}\right|\right)$ can be expressed as $\left|B_{r}\right|=\left|\phi_{o p e n}\right| / 4 \pi r^{2}$ at a heliocentric distance $r\left(r>>R_{\odot}\right)$. If $\theta_{H G}$ and $\phi_{H G}$ are the heliographic latitude and longitude, respectively, then the open magnetic flux can be derived by integrating $\left|B_{r}\left(R_{s s}, \theta_{H G}, \phi_{H G}\right)\right|$ over the surface of a sphere concentric to the Sun with radius $R_{s s}$, known as source surface. Using $\phi_{\text {open }}$, several studies have estimated $B_{r}$ at $r=1 \mathrm{AU}$ and compared it with the in situ near-Earth radial IMF intensity. The studies found a good correspondence between the estimated and observed values (Wang \& Sheeley, 1988, 1995; Wang et al., 2000; A. R. Yeates et al., 2010; Wang \& Sheeley Jr, 2015).

The present study aims to establish a connection between the MC's eroded magnetic flux and the IMF intensity. For achieving this we explore the relationship between annual averages of MC's eroded flux and the $\phi_{\text {open }}$ over two solar cycles. Also, we present a statistical analysis to investigate the geoeffectiveness of eroded MCs. We describe the data and their sources in section 2 and present our analysis methodology in section 3 . Results are presented in section 4 followed by discussion and conclusions in section 5 and section 6 , respectively.

\section{Instrumentation and Overview of Data}

We use 64s average level 2 (verified) data of merged interplanetary magnetic field and solar wind parameters (http://www.srl.caltech.edu/ACE/ASC/level2/lvl2DATA _MAG-SWEPAM.html) from the Advanced Composition Explorer (ACE) (Stone et al., 1998) spacecraft's Solar wind Electron, Proton and Alpha Monitor (SWEPAM) (McComas et al., 1998) and Magnetic Field Experiment (MAG) (C. W. Smith et al., 1998) instruments. The selected data is in Geocentric Solar Ecliptic (GSE) coordinate system and available since the year 1998. In GSE coordinate system $\hat{x}_{G S E}$ points toward the Sun, $\hat{z}_{G S E}$ points 
towards the north pole (of the ecliptic) and $\hat{y}_{G S E}$ completes the right-handed system. To obtain the geomagnetic response of $\mathrm{MC}$ we use the geomagnetic activity index (Dst) available at the World Data Center, Kyoto (http://wdc.kugi.kyoto-u.ac.jp/dstdir/ index.html) in a temporal resolution of 1 hour.

We obtain the radial magnetic field data from synoptic magnetograms (http:// hmi.stanford.edu/data/synoptic.html) collected by the Michelson Doppler Imager (MDI) (Scherrer et al., 1995) onboard the Solar and Heliospheric Observatory (SOHO) (Domingo et al., 1995) and the Helioseismic and Magnetic Imager (HMI) (Scherrer et al., 2012) onboard the Solar Dynamics Observatory (SDO) (Pesnell et al., 2012). The data from MDI and HMI is collected for a period of 1998-April 2010 and May 2010-2018, respectively. We utilize the radial magnetic field data to extrapolate the coronal magnetic field till the source surface at $r=R_{s s}=2.5 R_{\odot}$ using a global PFSS extrapolation model.

\subsection{MC events and criteria for selection}

In general, MCs are identified following the criteria defined by Burlaga et al. (1981) which are (1) throughout an MC interval the magnetic field intensity enhances (compared to the ambient solar wind), (2) smooth rotation in both $B_{y, G S E}$ and $B_{z, G S E}$ components exists, (3) the proton temperature falls below the expected solar wind temperature (Lopez \& Freeman, 1986), and (4) the plasma $\beta$ parameter is less than 1. For solar cycle 23 (19982007) we consider 37 MCs from a list of 109 MCs selected by Ruffenach et al. (2015). The study categorized the MCs as Q1 (both the front and rear boundaries of MCs are well determined), Q2 (any one of the two boundaries is identified without ambiguity) and Q3 (both the boundaries are difficult to identify). Of them, we select only Q1 and Q2 type MCs for our study as the fitting of flux ropes to the MCs is impacted by incorrect boundary selection. The events during 2008-2014 are obtained from the MC list published by Gopalswamy et al. (2015) and the events of 2015-2018 are selected from the Richardson and Cane (2010) ICME catalogue (http://www.srl.caltech.edu/ACE/ASC/ DATA/level3/icmetable2.htm) after manually identifying each of their boundaries. Thus, we select a total number of 35 events during solar cycle 24 . Note that, each of the events selected here is consistent with Burlaga et al. (1981)'s definition of MCs. For none of the events, the spacecraft crosses the MC flux ropes parallel to the legs. This is because those cases lead an azimuthal flux asymmetry which may be misinterpreted as a result of ero- 
sion (Ruffenach et al., 2015). Also, there exist uncertainties in fitting parameters [e.g. Marubashi and Lepping (2007); Lepping et al. (2003)] while those MCs are fit with the cylindrical flux rope model. Janvier et al. (2013) introduced a location angle $(\lambda)$ measured from the plane $\left(\hat{y}_{G S E}, \hat{z}_{G S E}\right)$ towards the MC axis. It is a proxy for the spacecraft crossing distance from the MC nose. The $\lambda$ evolves monotonically along the $\mathrm{MC}$ flux rope with $\lambda=-90^{\circ}$ in one leg, $\lambda=0^{\circ}$ at its nose, and $\lambda=90^{\circ}$ in the other leg. Ruffenach et al. (2015) estimated that errors in the azimuthal flux imbalance is less than $10 \%$ if $\lambda$ holds a value between $\pm 45^{\circ}$. Thus we study only those MCs which cross the spacecraft trajectory sufficiently close to their apex by selecting $\lambda<\left|45^{\circ}\right|$.

\section{Analysis methodology}

Several case studies (Dasso et al., 2006, 2007) show the presence of unbalanced azimuthal magnetic flux in MCs although MCs maintain a classical characteristic. Since the outer part of an MC is mainly affected by reconnection and the azimuthal flux is much greater than the axial flux in there (Ruffenach et al., 2015), the asymmetry is mainly observed in the MC's azimuthal magnetic flux. To estimate this asymmetry we employ the "direct method" developed by Dasso et al. (2006).

\subsection{Measurement of MC's eroded magnetic flux}

The direct method uses the principle of magnetic flux conservation $(\nabla \cdot \mathbf{B}=0)$ across the plane formed by the spacecraft trajectory and MC axis to determine and analyse the MC's accumulated azimuthal magnetic flux. The MC axis orientation is defined by a latitude angle $\theta$ (the angle between MC's axis and the ecliptic plane) varying from $-90^{\circ}$ to $90^{\circ}$ and a longitude angle $\phi$ (the angle between the projection of MC's axis on the ecliptic plane and the Earth-Sun direction) varying from $0^{\circ}$ to $360^{\circ}$. Using $\theta$ and $\phi$, we rotate the observed MC from GSE to the local MC coordinate defined by Dasso et al. (2006) and derive $\mathbf{B}_{\text {cloud }}$ and $\mathbf{V}_{\text {cloud }}$ from $\mathbf{B}_{G S E}$ and $\mathbf{V}_{G S E}$. To obtain $\theta$ and $\phi$ we use a constant- $\alpha$ force-free flux rope model (Burlaga, 1988; Marubashi, 1986) to leastsquare fit the observed MCs. The model follows the equation $\nabla \times \mathbf{B}=\alpha \mathbf{B}$, where $\alpha$ is a constant and allows self-similar expansion of an MC. The Lundquist's solution to the equation (Lepping et al., 1990) provides the modeled magnetic field vectors in the cylindrical coordinate system. After performing a series of iterations through proper adjustment of model parameters based on minimizing the difference between modeled and 
observed magnetic field vectors, a final set of best-fit parameters including $\theta$ and $\phi$ are ascertained (e.g, Marubashi and Lepping (2007); Marubashi et al. (2012)).

If $\theta, \phi$ and the time of $\mathrm{MC}$ front $\left(t_{\text {front }}\right)$ and rear boundaries $\left(t_{\text {rear }}\right)$ are known,

the accumulated azimuthal magnetic flux per unit length $\left(\frac{\phi_{y}\left(t_{1}, t_{2}\right)}{L}\right)$ can be measured using

$$
\frac{\phi_{y}\left(t_{1}, t_{2}\right)}{L}=\int_{t_{1}}^{t_{2}} B_{y, \text { cloud }}(t) V_{x, \text { cloud }} d t
$$

where $L$ is the total length of the MC axis till $1 \mathrm{AU}, B_{y, \text { cloud }}$ and $V_{x, \text { cloud }}$ are the MC's magnetic field and velocity components computed in the local cloud coordinate system, respectively. The times $t_{1}$ and $t_{2}$ represent generic times defining the boundaries of the integral. The normalized perpendicular distance between spacecraft trajectory and MC axis is called the impact parameter $\left(Y_{0}\right)$. The accumulated azimuthal flux is more precisely estimated when $Y_{0}$ is low. In Figure 2 we plot the time evolution of $B_{y, \text { cloud }}$ (blue curve) along with $\phi_{y} / L$ (red curve) of two MCs during their passage through the spacecraft. Figure 2a shows an MC that is eroded at the front edge. The parameter $\phi_{y} / L$ is calculated considering $t_{1}=t_{\text {front }}$ and $t_{2}=t_{\text {rear }}$. Due to erosion its accumulated azimuthal flux shows an excess at its rear. The total azimuthal flux per unit length $\left(\phi_{a z}\right)$ that was originally present in that MC (before erosion) is estimated by the unsigned value of $\phi_{y}\left(t_{c e n t e r}, t_{\text {rear }}\right) / L$. Here $t_{\text {center }}$ (shown in dashed-dotted black vertical lines in Figures $2 \mathrm{a}$ and $2 \mathrm{~b}$ ) is the time when $B_{y, \text { cloud }}$ changes its sign and results in a maximum value of unsigned $\phi_{y} / L$. The time $t_{c e n t e r}$ is the time when closest approach of the spacecraft to the MC axis is achieved. The eroded azimuthal flux (per unit length) of the MC is represented by the absolute value of $\phi_{y} / L$ at $t_{\text {rear }}$. The second MC (Figure $2 \mathrm{~b}$ ) is eroded at its rear and shows a flux imbalance at its front. Here $\phi_{y} / L$ is calculated considering $t_{1}=t_{\text {rear }}$ and $t_{2}=t_{\text {front }}$. For this case $\phi_{a z}$ is estimated by the unsigned value of $\phi_{y}\left(t_{\text {center }}, t_{\text {front }}\right) / L$. The eroded flux of the MC is defined by the absolute value of $\phi_{y} / L$ at $t_{\text {front }}$. We next define $\phi_{\text {erod }}$ as the eroded flux normalized to $\phi_{a z}$. The dashed-dotted green vertical lines show the estimated rear (Figure 2a) and front (Figure $2 \mathrm{~b}$ ) boundaries of the remaining flux-rope when it is observed in situ.

\subsection{Estimation of the Sun's open flux}

An estimate of the Sun's open flux is traditionally made based on potential field extrapolation of the Sun's coronal field (satisfying $\nabla \times \mathbf{B}=0$, Schatten et al. (1969)). 
Following van Ballegooijen et al. (2000) and using a module developed by A. Yeates (2018) we extrapolate the coronal magnetic field utilizing solar surface synoptic maps from MDI and HMI as the lower boundary condition. The unsigned open flux $\left(\left|\phi_{\text {open }}\right|\right)$ is estimated at $R_{s s}=2.5 R_{\odot}$. We obtain a strong positive correlation (with linear correlation coefficient, $r_{p}=0.89$ at $99.99 \%$ confidence level) between the yearly averaged IMF intensity observed in situ at $1 \mathrm{AU}$ and $\left|\phi_{\text {open }}\right|$. Therefore, $\left|\phi_{\text {open }}\right|$ can be used as a proxy of the IMF intensity.

\section{Analysis and Results}

By applying the direct method to all the selected MCs we derive $\phi_{a z}, \phi_{\text {erod }}$, and the position of the flux imbalance (front or back) for each of the MCs. In Figure 3 (a) we demonstrate the effect of MC's erosion on its geoeffectiveness by plotting MC's remaining azimuthal flux ( $\phi_{a z}-$ eroded flux) versus its geomagnetic response (quantified by $D s t_{m}$ - the minimum value of $D s t$ during an MC's passage). The correlation between the remaining flux and $D s t_{m}\left(r_{p}=-0.7\right.$ at $99.99 \%$ confidence) is slightly lower than the correlation between total flux and $D s t_{m}\left(r_{p}=-0.76\right.$ at $99.99 \%$ confidence $)$ if all events are considered. However, it is to be noted that the impact of MC's azimuthal flux erosion on its geomagnetic response is expected only if $B_{z, G S E}$ has a negative value at the reconnection site and the MC's azimuthal field component corresponds to $B_{z, G S E}$ (i.e., $\theta, \lambda \sim 0^{\circ}$ ) near the Earth (i.e., at $1 \mathrm{AU}$ ). Our sample has $9 \mathrm{MCs}$ (depicted by red dots in Figure 3 (a)) which have negative $B_{Z, G S E}$ at their reconnection sites and $|\theta|,|\lambda| \leq$ $30^{\circ}$ (i.e., close to the above conditions, while still providing reasonable sample size). For these events the correlation between the remaining flux of MCs and $D s t_{m}$ is higher $\left(r_{p}=\right.$ -0.95 at $99.99 \%$ confidence $)$ compared to that between $\phi_{a z}$ and $D s t_{m}\left(r_{p}=-0.88\right.$ at 99.99\% confidence). This reveals the impact of flux erosion on the geoeffectiveness of MCs.

We notice that out of $72,40 \mathrm{MCs}$ show the flux imbalance at rear with an average value of $\phi_{\text {erod }} \sim 0.26$ and $32 \mathrm{MCs}$ show flux imbalance at front with an average $\phi_{\text {erod }}$ $\sim 0.23$. The average eroded flux irrespective of the erosion position during solar cycle 23 and 24 are found to be 0.276 and 0.22 , respectively. Our result matches well with the value of normalized average eroded flux found by Ruffenach et al. (2015) for the events during solar cycle 23. MCs showing flux imbalance at the front or rear boundaries are named as $M C_{F}$ and $M C_{R}$, respectively. In Table 1, we show the number of total MCs (column 2), $M C_{R}$ (column 3), $M C_{F}$ (column 4), annual averages of $\phi_{\text {erod }}$ (column 5), 
and unsigned $\phi_{\text {open }}$ (column 6) with their standard deviations corresponding to each year mentioned in column 1 .

\subsection{Comparison between the Sun's unsigned open flux and MC flux ero- sion}

The erosion of magnetic flux in an MC depends on the rate of reconnection between the magnetic field of the MC and IMF. As it is stated before that the reconnection rate scales with the IMF intensity, a correlation is expected between $\phi_{\text {erod }}$ and the prevailing IMF strength whose proxy is the unsigned $\phi_{\text {open }}$. We define the yearly average of the normalized eroded azimuthal flux $\left(\bar{\phi}_{\text {erod }}\right)$ by averaging over $\phi_{\text {erod }}$ with the total number of events $\left(N_{M C}\right)$ in each year. In Figure $3 \mathrm{~b}$, we plot $\bar{\phi}_{\text {erod }}$ and the yearly average of unsigned $\phi_{\text {open }}\left(\left|\bar{\phi}_{\text {open }}\right|\right)$ in solid red and dashed black curves, respectively, along with error bars over the period encompassing solar cycle 23 and 24 . The error bars are calculated by estimating, $\frac{\sigma}{\sqrt{N_{\text {event }}}}$, where $\sigma$ is the standard deviation and $N_{\text {event }}$ represents number of MC events for the eroded flux and number of PFSS extrapolations for the calculation of the open flux in each year. A correlation study is performed between $\bar{\phi}_{\text {erod }}$ and $\left|\bar{\phi}_{\text {open }}\right|$. We obtain a Pearson correlation coefficient $\left(r_{p}\right)$ of 0.56 and a Spearman's rank correlation coefficient $\left(r_{s}\right)$ of 0.44 , at $99 \%$ and $95 \%$ confidence levels, respectively. To confirm our result we compute $r_{p}$ including the parameters' error bar information and find the value of $r_{p}$ as $0.51 \pm 0.03$ at $98 \%$ confidence level. In Figure $3 \mathrm{c}$, we show a scat-

ter plot between $\bar{\phi}_{\text {erod }}$ and $\left|\bar{\phi}_{\text {open }}\right|$. The observed correspondence between the eroded flux and the Sun's open flux indicates an underlying linear relationship between the reconnection rate and the IMF intensity. To relate these two parameters, we perform a leastsquares fit to $\left|\bar{\phi}_{\text {open }}\right|$ versus $\bar{\phi}_{\text {erod }}$ which gives the following equation

$$
\bar{\phi}_{\text {erod }}=0.06\left|\bar{\phi}_{\text {open }}\right|+0.06
$$

where $\left|\bar{\phi}_{\text {open }}\right|$ is in units of $10^{22} M x$. The solid blue line over plotted on the scatter plot represents the least-squares fitting.

\section{Discussion}

To establish a correspondence between MC's eroded flux and the IMF intensity, we study the dependency of the MC's eroded flux on the Sun's open flux over two solar cycles. We find that about $44 \%$ of the total MCs studied here shows an imbalance 
at their rear boundaries. A numerical simulation of reconnection between an MC and solar wind performed by Schmidt and Cargill (2003) shows an enhancement in reconnection with the increasing relative speed of the MC compared to the solar wind. If an $\mathrm{MC}$ is followed and compressed by a fast solar wind, reconnection may occur at the MC's rear boundary. Such high speed solar wind primarily originates from polar coronal holes and may extend towards low latitudes (Fenrich \& Luhmann, 1998). Also, MCs can be compressed by co-rotating interaction regions whose formation is related to the presence of coronal holes (Rouillard et al., 2010).

In our study we obtain the orientation of an MC axis by fitting the observed MC using a force free cylindrical flux rope model. The model is impacted by several factors, such as, incorrect boundary selection (Lepping et al., 2003), non circular MC cross section (Savani et al., 2011b, 2011a) and high impact parameter values (Démoulin et al., 2013; Riley et al., 2004). Ruffenach et al. (2015) showed that the flux rope fitting method has a tendency of deriving a consistently lower value of eroded flux because the model is based on axisymmetric geometry. Also, a high impact parameter lowers the total azimuthal magnetic flux that causes an overestimation of the normalized eroded flux.

The imbalance in MC azimuthal flux accumulated along the spacecraft path may occur due to various other reasons. When a high-speed CME propagates from Sun to Earth it undergoes strong deceleration (Jones et al., 2007). If a cool, dense filament material is located at the base of the CME, the momentum of the filament results in its forward movement through the decelerating CME. Thus, the protruding filament drives flow leading to a sideways transport of the CME's poloidal field. This can generate an azimuthal flux imbalance in the CME flux rope by the time the CME reaches the Earth. Manchester et al. (2014) explained such flux imbalances through simulation of a threepart structure CME observed on 20 January 2005. In such cases, the eroded magnetic flux amount does not depend on the rate of reconnection between the MC and the IMF. This may also contribute to a lower correlation between the amount of eroded MC flux and $\phi_{\text {open }}$. Nonetheless, we find the correlation to be significant and the underlying relationship between $\phi_{\text {erod }}$ and $\phi_{\text {open }}$ to be linear. 


\section{Conclusions}

We establish a relationship between the azimuthal magnetic flux erosion of MCs and the Sun's unsigned open flux that regulates the IMF intensity. Utilizing the direct method we investigate $72 \mathrm{MCs}$ spanning solar cycles 23 and 24 to estimate the magnetic flux imbalance and erosion. We find that on average $28 \%$ and $22 \%$ of the total azimuthal magnetic flux of MCs of solar cycle 23 and 24, respectively, are eroded during their propagation through the inner heliosphere. The reconnection causing this erosion may occur on either side of the MC boundaries and peel off an almost similar amount of magnetic flux. We compare the annual averages of solar open flux to the average fraction of the eroded magnetic flux of MCs over the past 21 years (from 1998 to 2018) and find a significant positive correlation with an underlying linear relationship. Since the solar open flux is strongly correlated with the IMF intensity, this is suggestive of the latter's role in MC flux erosion.

We note that the solar open flux is governed by the emergence and redistribution of active region magnetic fields on the Sun's surface due to surface flux transport processes - a crucial component of the solar dynamo mechanism (Bhowmik \& Nandy, 2018). Given that the solar wind dispersed open flux determines the ambient heliospheric magnetic field (IMF), the latter's solar cycle modulation provides a novel pathway via which the large-scale solar cycle can govern flux erosion and thus possibly the geoeffectiveness of interplanetary magnetic clouds.

\section{Acknowledgments}

The Center of Excellence in Space Sciences India (CESSI) is funded by the Ministry of Human Resource Development, Government of India under the Frontier Areas of Science and Technology scheme. S.D. acknowledges funding from the DST-INSPIRE program of the Government of India. The authors are grateful to Benoit Lavraud for insightful discussions and suggestions. We thank Katsuhide Marubashi for making available the constant- $\alpha$ force free cylindrical flux rope model. We thank an anonymous referee for useful comments. The authors acknowledge the use of data from the SDO/HMI, SOHO/MDI, ACE instruments, and the World Data Center for Geomagnetism, Kyoto. 


\section{References}

Alksne, A. Y., \& Webster, D. L. (1970). Magnetic and electric fields in the magnetosheath. Planetary and Space Science, 18(8), 1203-1212.

Balogh, A., Smith, E. J., Tsurutani, B. T., Southwood, D. J., Forsyth, R. J., \& Horbury, T. S. (1995, May). The Heliospheric Magnetic Field Over the South Polar Region of the Sun. Science, 268, 1007-1010. doi: 10.1126/ science.268.5213.1007

Bhowmik, P., \& Nandy, D. (2018, Dec). Prediction of the strength and timing of sunspot cycle 25 reveal decadal-scale space environmental conditions. Nature Communications, 9, 5209. doi: 10.1038/s41467-018-07690-0

Birn, J., Drake, J. F., Shay, M. A., Rogers, B. N., Denton, R. E., Hesse, M., ... Pritchett, P. L. (2001, March). Geospace Environmental Modeling (GEM) magnetic reconnection challenge. J. Geophys.Res. Space Physics, 106, 37153720. doi: 10.1029/1999JA900449

Burlaga, L. F. (1988, July). Magnetic clouds and force-free fields with constant alpha. J. Geophys.Res. Space Physics, 93, 7217-7224. doi: 10.1029/ JA093iA07p07217

Burlaga, L. F., Hundhausen, A. J., \& Zhao, X.-P. (1981, October). The coronal and interplanetary current sheet in early 1976. J. Geophys.Res. Space Physics, 86, 8893-8898. doi: 10.1029/JA086iA11p08893

Cassak, P. A., \& Shay, M. A. (2007, October). Scaling of asymmetric magnetic reconnection: General theory and collisional simulations. Physics of Plasmas, 14(10), 102114. doi: 10.1063/1.2795630

Crooker, N., Luhmann, J., Russell, C., Smith, E., Spreiter, J., \& Stahara, S. (1985). Magnetic field draping against the dayside magnetopause. $\quad J$. Geophys.Res. Space Physics, 90(A4), 3505-3510.

Dasso, S., Mandrini, C. H., Démoulin, P., \& Luoni, M. L. (2006, August). A new model-independent method to compute magnetic helicity in magnetic clouds. Astron. Astrophys., 455, 349-359. doi: 10.1051/0004-6361:20064806

Dasso, S., Nakwacki, M. S., Démoulin, P., \& Mandrini, C. H. (2007, August). Progressive Transformation of a Flux Rope to an ICME. Comparative Analysis Using the Direct and Fitted Expansion Methods. Sol. Phys., 244, 115-137. doi: $10.1007 / \mathrm{s} 11207-007-9034-2$ 
Démoulin, P., Dasso, S., \& Janvier, M. (2013, Feb). Does spacecraft trajectory strongly affect detection of magnetic clouds? Astron. Astrophys., 550, A3. doi: $10.1051 / 0004-6361 / 201220535$

Domingo, V., Fleck, B., \& Poland, A. I. (1995, December). The SOHO Mission: an Overview. Sol. Phys., 162, 1-37. doi: 10.1007/BF00733425

Erdős, G., \& Balogh, A. (2014, January). Magnetic Flux Density in the Heliosphere through Several Solar Cycles. Astrophys. J., 781, 50. doi: 10.1088/0004-637X/ $781 / 1 / 50$

Fenrich, F. R., \& Luhmann, J. G. (1998). Geomagnetic response to magnetic clouds of different polarity. Geophys. Res. Lett., 25, 2999-3002. doi: 10.1029/98GL51180

Goldstein, H. (1983, November). On the field configuration in magnetic clouds. In Nasa conference publication (Vol. 228).

Gopalswamy, N., Yashiro, S., Xie, H., Akiyama, S., \& Mäkelä, P. 2015, November). $\quad$ Properties and geoeffectiveness of magnetic clouds during solar cycles 23 and 24. J. Geophys.Res. Space Physics, 120, 9221-9245. doi: 10.1002/2015JA021446

Gosling, J. T., \& McComas, D. J. (1987, April). Field line draping about fast coronal mass ejecta - A source of strong out-of-the-ecliptic interplanetary magnetic fields. Geophys. Res. Lett., 14, 355-358. doi: 10.1029/GL014i004p00355

Janvier, M., Démoulin, P., \& Dasso, S. (2013, Aug). Global axis shape of magnetic clouds deduced from the distribution of their local axis orientation. $A s$ tron. Astrophys., 556, A50. doi: 10.1051/0004-6361/201321442

Jones, R. A., Breen, A. R., Fallows, R. A., Canals, A., Bisi, M. M., \& Lawrence, G. (2007, Aug). Interaction between coronal mass ejections and the solar wind. Journal of Geophysical Research (Space Physics), 112(A8), A08107. doi: 10.1029/2006JA011875

Klein, L. W., \& Burlaga, L. F. ～(1982, February). Interplanetary magnetic clouds at 1 AU. $\quad$ J. Geophys.Res. Space Physics, 87, 613-624. doi: 10.1029/JA087iA02p00613

Lavraud, B., \& Borovsky, J. E. $\quad$ (2008, September). Altered solar windmagnetosphere interaction at low Mach numbers: Coronal mass ejections.

J. Geophys.Res. Space Physics, 113, A00B08. doi: 10.1029/2008JA013192 
Lavraud, B., Ruffenach, A., Rouillard, A. P., Kajdic, P., Manchester, W. B., \& Lugaz, N. (2014, January). Geo-effectiveness and radial dependence of magnetic cloud erosion by magnetic reconnection. J. Geophys.Res. Space Physics, 119, 26-35. doi: 10.1002/2013JA019154

Lepping, R. P., Berdichevsky, D. B., \& Ferguson, T. J. (2003, October). Estimated errors in magnetic cloud model fit parameters with force-free cylindrically symmetric assumptions. J. Geophys.Res. Space Physics, 108, 1356. doi: 10.1029/2002JA009657

Lepping, R. P., Jones, J. A., \& Burlaga, L. F. ～(1990, August). Magnetic field structure of interplanetary magnetic clouds at 1 AU. J. Geophys.Res. Space Physics, 95, 11957-11965. doi: 10.1029/JA095iA08p11957

Lopez, R. E., \& Freeman, J. W. (1986). Solar wind proton temperature-velocity relationship. J. Geophys.Res. Space Physics, 91(A2), 1701-1705.

Manchester, W. B., Kozyra, J. U., Lepri, S. T., \& Lavraud, B. (2014, July). Simulation of magnetic cloud erosion during propagation. J. Geophys.Res. Space Physics, 119, 5449-5464. doi: 10.1002/2014JA019882

Marubashi, K. (1986). Structure of the interplanetary magnetic clouds and their solar origins. $\quad$ Advances in Space Research, 6, 335-338. doi: 10.1016/0273 $-1177(86) 90172-9$

Marubashi, K., Cho, K.-S., Kim, Y.-H., Park, Y.-D., \& Park, S.-H. (2012, January). Geometry of the 20 November 2003 magnetic cloud. J. Geophys.Res. Space Physics, 117, A01101. doi: 10.1029/2011JA016802

Marubashi, K., \& Lepping, R. P. (2007, November). Long-duration magnetic clouds: a comparison of analyses using torus- and cylinder-shaped flux rope models. Annales Geophysicae, 25, 2453-2477. doi: 10.5194/angeo-25-2453-2007

McComas, D. J., Bame, S. J., Barker, P., Feldman, W. C., Phillips, J. L., Riley, P., \& Griffee, J. W. (1998, July). Solar Wind Electron Proton Alpha Monitor (SWEPAM) for the Advanced Composition Explorer. Space Sci. Rev., 86, 563-612. doi: 10.1023/A:1005040232597

McComas, D. J., Gosling, J. T., Winterhalter, D., \& Smith, E. J. ～(1988, April). Interplanetary magnetic field draping about fast coronal mass ejecta in the outer heliosphere. J. Geophys.Res. Space Physics, 93, 2519-2526. doi: 10.1029/JA093iA04p02519 
Möstl, C., Miklenic, C., Farrugia, C. J., Temmer, M., Veronig, A., Galvin, A. B., ... Biernat, H. K. (2008, October). Two-spacecraft reconstruction of a magnetic cloud and comparison to its solar source. Annales Geophysicae, 26, 3139-3152. doi: 10.5194/angeo-26-3139-2008

Nakamura, T. K. M., Genestreti, K. J., Liu, Y. H., Nakamura, R., Teh, W. L., Hasegawa, H., .. Giles, B. L. (2018, Nov). Measurement of the Magnetic Reconnection Rate in the Earth's Magnetotail. Journal of Geophysical Research (Space Physics), 123(11), 9150-9168. doi: 10.1029/2018JA025713

Pal, S., Gopalswamy, N., Nandy, D., Akiyama, S., Yashiro, S., Makela, P., \& Xie, H. (2017, Dec). A Sun-to-Earth Analysis of Magnetic Helicity of the 2013 March 17-18 Interplanetary Coronal Mass Ejection. Astrophys. J., 851(2), 123 . doi: $10.3847 / 1538-4357 /$ aa9983

Parker, E. N. (1957, Dec). Sweet's Mechanism for Merging Magnetic Fields in Conducting Fluids. Journal of Geophysical Research, 62(4), 509-520. doi: 10.1029/ JZ062i004p00509

Parker, E. N. (1973, February). The Reconnection Rate of Magnetic Fields. Astrophys. J., 180, 247-252. doi: 10.1086/151959

Pesnell, W. D., Thompson, B. J., \& Chamberlin, P. C. (2012, January). The Solar Dynamics Observatory (SDO). Sol. Phys., 275, 3-15. doi: 10.1007/s11207-011 $-9841-3$

Richardson, I. G., \& Cane, H. V. (2010, Jun 01). Near-earth interplanetary coronal mass ejections during solar cycle 23 (1996-2009): Catalog and summary of properties. Sol. Phys., 264(1), 189-237. Retrieved from https://doi.org/ 10.1007/s11207-010-9568-6 doi: 10.1007/s11207-010-9568-6

Riley, P., Linker, J. A., Lionello, R., Mikić, Z., Odstrcil, D., Hidalgo, M. A., ... Lynch, B. J. (2004, Oct). Fitting flux ropes to a global MHD solution: a comparison of techniques. Journal of Atmospheric and Solar-Terrestrial Physics, 66(15-16), 1321-1331. doi: 10.1016/j.jastp.2004.03.019

Rouillard, A. P., Lavraud, B., Sheeley, N. R., Davies, J. A., Burlaga, L. F., Savani, N. P., .. Forsyth, R. J. (2010, August). White Light and In Situ Comparison of a Forming Merged Interaction Region. Astrophys. J., 719, 1385-1392. doi: 10.1088/0004-637X/719/2/1385

Ruffenach, A., Lavraud, B., Farrugia, C. J., Démoulin, P., Dasso, S., Owens, M. J., 
... Galvin, A. B. (2015, January). Statistical study of magnetic cloud erosion by magnetic reconnection. J. Geophys.Res. Space Physics, 120, 43-60. doi: 10.1002/2014JA020628

Ruffenach, A., Lavraud, B., Owens, M. J., Sauvaud, J.-A., Savani, N. P., Rouillard, A. P., ... Galvin, A. B. (2012, September). Multispacecraft observation of magnetic cloud erosion by magnetic reconnection during propagation. $J$. Geophys.Res. Space Physics, 117, A09101. doi: 10.1029/2012JA017624

Savani, N. P., Owens, M. J., Rouillard, A. P., Forsyth, R. J., Kusano, K., Shiota, D., \& Kataoka, R. (2011a, Apr). Evolution of Coronal Mass Ejection Morphology with Increasing Heliocentric Distance. I. Geometrical Analysis. Astrophys. J., 731 (2), 109. doi: 10.1088/0004-637X/731/2/109

Savani, N. P., Owens, M. J., Rouillard, A. P., Forsyth, R. J., Kusano, K., Shiota, D., ... Bothmer, V. (2011b, May). Evolution of Coronal Mass Ejection Morphology with Increasing Heliocentric Distance. II. In Situ Observations. Astrophys. J., 732(2), 117. doi: 10.1088/0004-637X/732/2/117

Schatten, K. H., Wilcox, J. M., \& Ness, N. F. (1969, Mar 01). A model of interplanetary and coronal magnetic fields. Sol. Phys., 6(3), 442-455. Retrieved from https://doi.org/10.1007/BF00146478 doi: 10.1007/BF00146478

Scherrer, P. H., Bogart, R. S., Bush, R. I., Hoeksema, J. T., Kosovichev, A. G., Schou, J., ... MDI Engineering Team (1995, December). The Solar Oscillations Investigation - Michelson Doppler Imager. Sol. Phys., 162, 129-188. doi: $10.1007 / \mathrm{BF} 00733429$

Scherrer, P. H., Schou, J., Bush, R. I., Kosovichev, A. G., Bogart, R. S., Hoeksema, J. T., .. Tomczyk, S. (2012, January). The Helioseismic and Magnetic Imager (HMI) Investigation for the Solar Dynamics Observatory (SDO). Sol. Phys., 275, 207-227. doi: 10.1007/s11207-011-9834-2

Schmidt, J. M., \& Cargill, P. J. (2003, January). Magnetic reconnection between a magnetic cloud and the solar wind magnetic field. J. Geophys.Res. Space Physics, 108, 1023. doi: 10.1029/2002JA009325

Smith, C. W., L'Heureux, J., Ness, N. F., Acuña, M. H., Burlaga, L. F., \& Scheifele, J. (1998, July). The ACE Magnetic Fields Experiment. Space Sci. Rev., 86, 613-632. doi: 10.1023/A:1005092216668

Smith, E. J., \& Balogh, A. (2008, November). Decrease in heliospheric magnetic 
flux in this solar minimum: Recent Ulysses magnetic field observations. Geophys. Res. Lett., 35, L22103. doi: 10.1029/2008GL035345

Stone, E. C., Frandsen, A. M., Mewaldt, R. A., Christian, E. R., Margolies, D., Ormes, J. F., \& Snow, F. (1998, July). The Advanced Composition Explorer. Space Sci. Rev., 86, 1-22. doi: 10.1023/A:1005082526237

Sweet, P. A. (1958). 14. the neutral point theory of solar flares. Symposium - International Astronomical Union, 6, 123-134. doi: 10.1017/S0074180900237704

Taubenschuss, U., Erkaev, N. V., Biernat, H. K., Farrugia, C. J., Möstl, C., \& Amerstorfer, U. V. (2010). The role of magnetic handedness in magnetic cloud propagation. Annales Geophysicae, 28(5), 1075-1100. Retrieved from https:// www. ann-geophys.net/28/1075/2010/ doi: 10.5194/angeo-28-1075-2010

van Ballegooijen, A. A., Priest, E. R., \& Mackay, D. H. $\quad$ (2000, aug). Mean field model for the formation of filament channels on the sun. Astrophys. J., 539(2), 983-994. Retrieved from https://doi.org/10.1086\%2F309265 doi: 10.1086/ 309265

Wang, Y.-M., Hawley, S. H., \& Sheeley, N. R. (1996). The magnetic nature of coronal holes. $\quad$ Science, 271(5248), 464-469. Retrieved from https://science.sciencemag.org/content/271/5248/464 doi: 10.1126/ science.271.5248.464

Wang, Y.-M., Lean, J., \& Sheeley, N. (2000). The long-term variation of the sun's open magnetic flux. Geophys. Res. Lett., 27(4), 505-508.

Wang, Y.-M., \& Sheeley, N. R., Jr. (1988, October). The solar origin of long-term variations of the interplanetary magnetic field strength. J. Geophys.Res. Space Physics, 93, 11227-11236. doi: 10.1029/JA093iA10p11227

Wang, Y.-M., \& Sheeley, N. R., Jr. (1995, July). Solar Implications of ULYSSES Interplanetary Field Measurements. Astrophys. J. Lett., 447, L143. doi: 10.1086/ 309578

Wang, Y.-M., \& Sheeley Jr, N. (2015). Coronal mass ejections and the solar cycle variation of the sun's open flux. Astrophys. J. Lett., 809(2), L24.

Yeates, A. (2018, October). antyeates1983/pfss: First release of pfss code. Retrieved from https://doi.org/10.5281/zenodo.1472183 doi: 10.5281/ zenodo. 1472183

Yeates, A. R., Mackay, D. H., van Ballegooijen, A. A., \& Constable, J. A. (2010). A 
nonpotential model for the sun's open magnetic flux.

J. Geophys.Res. Space

Physics, 115(A9). Retrieved from https://agupubs.onlinelibrary.wiley .com/doi/abs/10.1029/2010JA015611 doi: 10.1029/2010JA015611

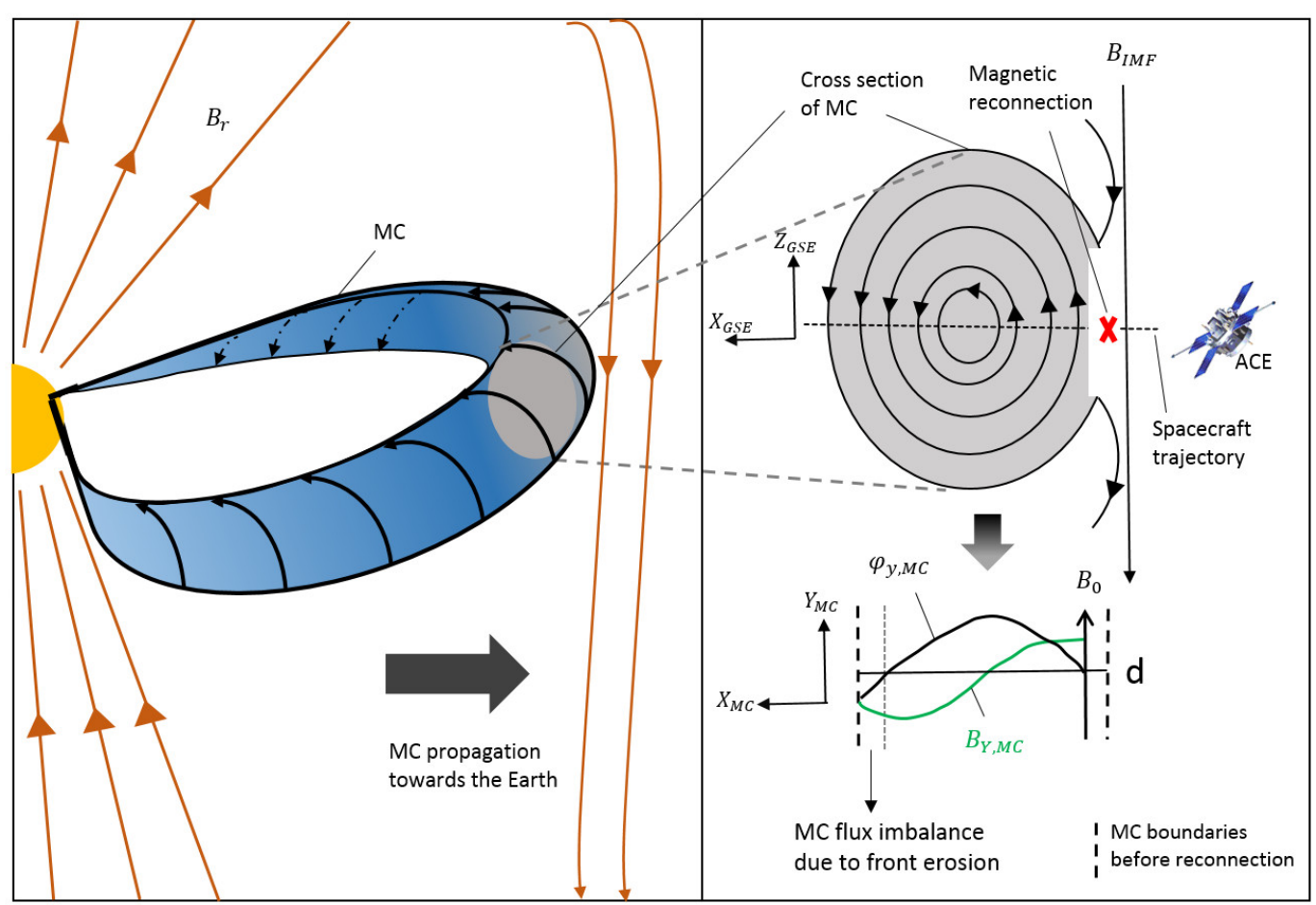

Figure 1. An idealized schematic of the ambient IMF draping about an MC propagating radially outward from the Sun towards the Earth (left), the MC's magnetic structure in a plane perpendicular to the ecliptic plane (right-top) and the expected variation in the MC's accumulated azimuthal flux due to its reconnection with the draped ambient IMF (right-bottom) 

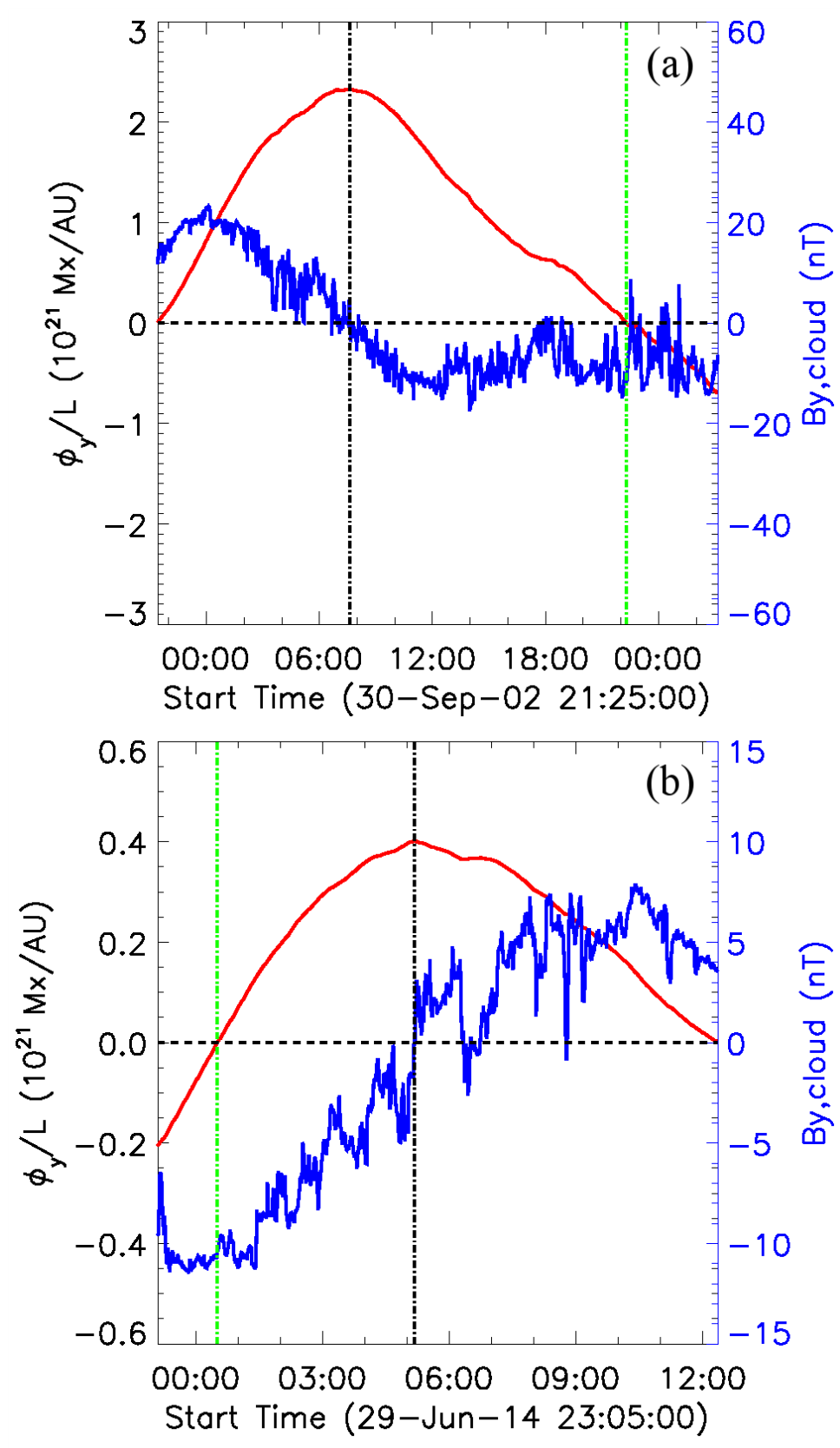

Figure 2. Plots depicting $B_{y, \text { cloud }}$ (blue) and $\phi_{y} / L$ (red) variations of two MCs during their passage past the spacecraft. The black and green vertical dashed-dotted lines represent $t_{c e n t e r}$ and the zero crossing of $\phi_{y} / L$ curves, respectively. (a) For an event where the flux erosion is at the MC-front. Here $\phi_{a z}=3.02 \times 10^{21} \mathrm{Mx} / \mathrm{AU}$ and eroded flux $=6.98 \times 10^{20} \mathrm{Mx} / \mathrm{AU}$. (b) For an event where the flux erosion is at MC-rear. Here $\phi_{a z}=6.06 \times 10^{20} \mathrm{Mx} / \mathrm{AU}$ and eroded flux $=$ $2.04 \times 10^{20} \mathrm{Mx} / \mathrm{AU}$. 

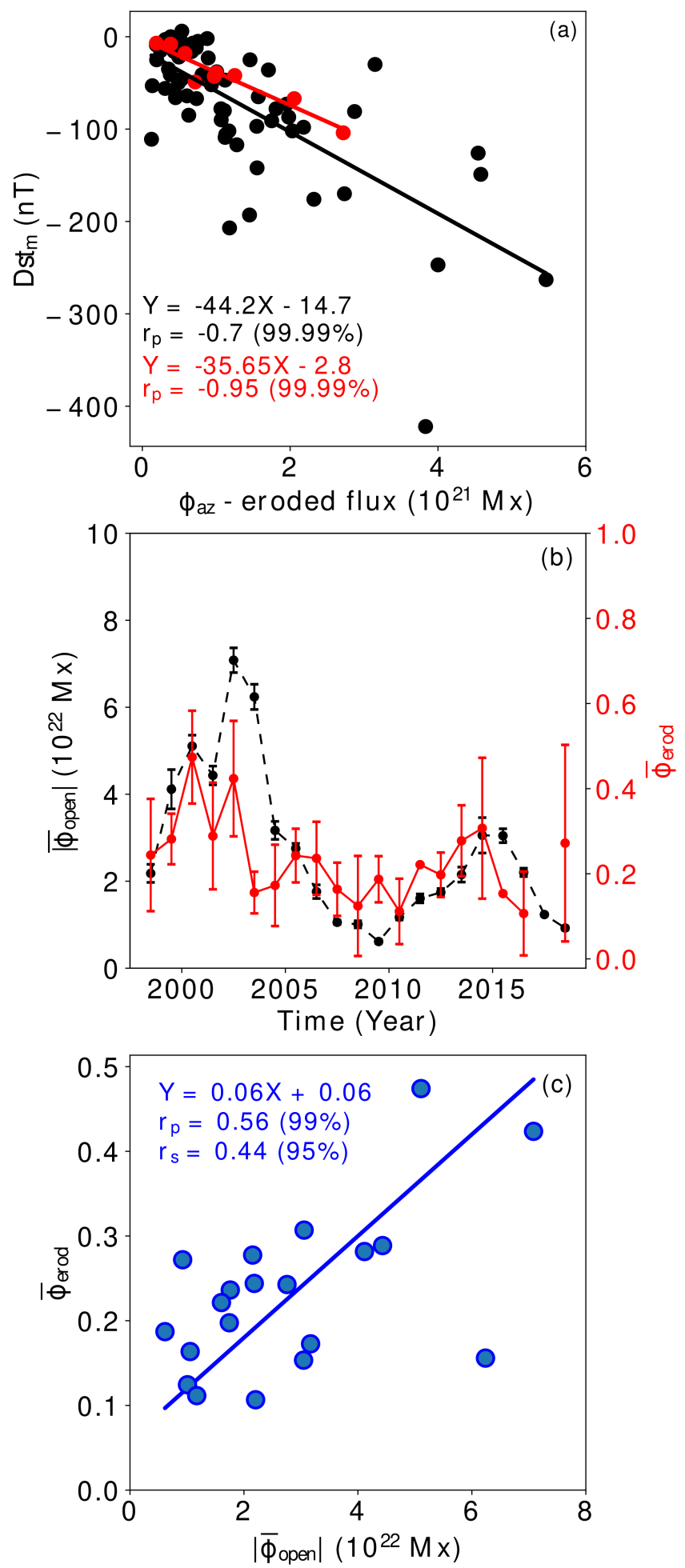

Figure 3. (a) Scatter plot between the remaining flux of MCs and their geoeffectivness as quantified by the minimum $D s t$ index $\left(D s t_{m}\right)$. Least-squares fit considering all events (red and black dots) and the correlation are shown in black. For a subset of events (red dots only) as described in the text, the corresponding least-squares fit and correlation are depicted in red. (b) $\bar{\phi}_{\text {erod }}$ and $\left|\bar{\phi}_{\text {open }}\right|$ plotted against each year for solar cycle 23 and 24 in continuous red and dashed black curves, respectively. Vertical lines in corresponding colors represent the error bars for $\bar{\phi}_{\text {erod }}$ and $\left|\bar{\phi}_{\text {open }}\right|$. (c) Scatter plot of $\left|\bar{\phi}_{\text {open }}\right|$ versus $\bar{\phi}_{\text {erod }}$. The over plotted blue solid line shows the 